\title{
Correction to: An analysis of the radiological factors associated with respiratory failure in COVID-19 pneumonia and the CT features among different age categories
}

\author{
Akito Fukuda ${ }^{1,2} \cdot$ Noriyo Yanagawa $^{3} \cdot$ Noritaka Sekiya $^{4}\left(\mathbb{D} \cdot\right.$ Koji Ohyama $^{1} \cdot$ Makiko Yomota $^{2} \cdot$ Takanori Inui $^{3}$. \\ Sho Fujiwara ${ }^{1} \cdot$ Shoko Kawai $^{2} \cdot$ Kazuaki Fukushima $^{1} \cdot$ Masaru Tanaka $^{1} \cdot$ Taiichiro Kobayashi $^{1} \cdot$ Keishiro Yajima $^{1}$. \\ Akifumi Imamura'
}

Published online: 13 August 2021

(C) Japan Radiological Society 2021

\section{Correction to: \\ Japanese Journal of Radiology (2021) 39:783-790 https://doi.org/10.1007/s11604-021-01118-4}

In the original publication, the second author Noriyo Yanagawa should be treated as co-first author and the article note should be included as given in this Correction.

Also, the affiliation of sixth author: Takanori Inui, should be: Department of Radiology, Tokyo Metropolitan Cancer and Infectious Diseases Center Komagome Hospital, Tokyo, Japan, as listed in this Correction.
Publisher's Note Springer Nature remains neutral with regard to jurisdictional claims in published maps and institutional affiliations.

Akito Fukuda and Noriyo Yanagawa have equally contributed to this work and should be treated as first authors.

The original article can be found online at https://doi.org/10.1007/ s11604-021-01118-4.

Noritaka Sekiya qnmnk410@ybb.ne.jp

1 Department of Infectious Diseases, Tokyo Metropolitan Cancer and Infectious Diseases Center Komagome Hospital, Tokyo, Japan

2 Department of Respiratory Medicine, Tokyo Metropolitan Cancer and Infectious Diseases Center Komagome Hospital, Tokyo, Japan

3 Department of Radiology, Tokyo Metropolitan Cancer and Infectious Diseases Center Komagome Hospital, Tokyo, Japan

4 Department of Infection Prevention and Control, Department of Clinical Laboratory, Tokyo Metropolitan Cancer and Infectious Diseases Center Komagome Hospital, 3-18-22 Hon-komagome, Bunkyo-ku, Tokyo 1138677, Japan 